\title{
To PIOPED, or Not to PIOPED
}

F or the last 15 years, the development of lung scintigraphy has been closely connected with the PIOPED studies. In the latest analysis based on the PIOPED II data, published in this issue of The Journal of Nuclear Medicine (1), we learn that chest radiography may safely be substituted for the ventilation scan. However, I have to admit that I am not completely convinced of the usefulness of the PIOPED system itself. In fact, I am of the opinion that the PIOPED classification, with all its nondiagnostic cases, its intermediate-probability class, and its unfavorable complexity, led to the decline of ventilation-perfusion (V/Q) scintigraphy that we are experiencing

\section{See page 1741}

today. Furthermore I believe-and I state this with deep regret-that in the medium term the method might be lost to nuclear medicine. The reason is quite simple: if you tell a referring physician twice in a row that the patient he sent has a probability for pulmonary embolism of between $20 \%$ and $80 \%$, you will never be asked again — and rightly so. As far as CT angiography (CTA) is concerned, I am quite sure that a substantial number of examinations yield ambivalent results, especially on the segmental and subsegmental levels. Furthermore, from my own studies I know that CTA leads to a small number of false-positive diagnoses and a substantial number of false-negative diagnoses (2). The PIOPED II investigators came to the

Received Jun. 1, 2008; revision accepted Jul. 15, 2008.

For correspondence or reprints contact: Patrick Reinartz, Department of Nuclear Medicine, Radios-Clinic for Radiology and Nuclear Medicine, Juelicher Strasse 75, 40477 Duesseldorf, Germany. E-mail: reinartz@arcor.de

COPYRIGHT ( 2008 by the Society of Nuclear Medicine, Inc.

DOI: 10.2967/jnumed.108.053959 same results, finding a sensitivity and specificity for CTA of $83 \%$ and $96 \%$, respectively (3). Still, neither the PIOPED investigators nor their colleagues from the radiology departments concluded from these data that the development of CTA-probability classes is in order. Lung scintigraphy, if done properly, is a highly sensitive test that leads to a definitive diagnosis in about $97 \%$ and a correct diagnosis in over $90 \%$ of patients (4). The ventilation scan is easy to obtain and produces a low radiation exposure, and I cannot think of one reason why I should set it aside. However, I am from Europe, where Technegas (Cyclomedica) has been available for over 10 years now, which makes the procedure swift, clean, and easy.

The second aspect that amazes me about the PIOPED investigators is their ongoing and systematic neglect of the SPECT technique. SPECT acquisitions are neither hard to get nor complicated, expensive, or time consuming. The technique has proven its value in all clinical fields of nuclear medicine. I would very much like to see the reaction of the scientific and clinical community to an article in the $J N M$ proposing planar acquisitions for myocardial scintigraphy or brain scans. However, the designers of the PIOPED II trial seem to think that SPECT is negligible in the diagnosis of pulmonary embolism. At the same time, they demand that the "decline of V/Q use" has to be averted by increasing the value of the examination. Using state-of-theart techniques would, at least in my opinion, indeed increase the value of the lung scan. By disregarding SPECT, the whole comparison between CTA and V/Q scintigraphy becomes unbalanced and biased. Although CTA is done in present-day techniques using all available modern developments, the procedure of the perfusion scan has barely changed between PIOPED I and
PIOPED II. Thereby, a modern tomographic technique is compared with an outdated planar one. However, the PIOPED team sticks to conventional planar scans. The fact that no one complains about this fuzzy study design underlines the decline of V/Q scintigraphy. Why bother?

As a major conclusion of the article, we learn that "ventilation scans are superfluous in most patients." However, this statement implies that the physicians reading the perfusion scans are also familiar with interpreting chest radiographs with high accuracy. Among others, the chest radiography criteria mentioned in the article comprise "size and shape of the heart and hilar arteries, position of the diaphragm, presence or absence of pulmonary parenchymal abnormalities (consolidation, atelectasis, oligemia, edema), and pleural effusion." In addition, "pulmonary consolidations were considered suggestive of infarction if they had a semicircular or half-spindle shape and were arranged peripherally along the pleural surface." Although I am quite sure that most colleagues working in the field of nuclear medicine are well versed in reading ventilation scans, I very much doubt that they show the same level of expertise in interpreting chest radiographs.

The aspect of radiation exposure is discussed somewhat ambivalently. Although on the one hand the authors are highly motivated to reduce the patients' radiation exposure by substituting the ventilation scan with chest radiography, on the other hand they have absolutely no misgivings about advocating a broad use of CTA in the diagnosis of pulmonary embolism. At this point, we have to remind ourselves that the radiation exposure of the ventilation scan is rather small whereas that of CTA is quite substantial. According to the as-lowas-reasonably-achievable (ALARA) principle, the diagnostic test with the lowest 
reasonably achievable radiation exposure should be used, at least if it shows an acceptable accuracy. At present, the largest share of medically induced radiation is due to CT, and this development is aggravated by the new scanner generations with 32 or more detector rows. According to current epidemiologic studies, CT examinations will lead to a substantial number of patients with radiation-induced cancer $(5,6)$. To make things worse, the radiation exposure of CTA nearly triples when not only the chest but also the lower extremities are scanned. To sum it up: radiation exposure is a problem of CTA but not of lung scintigraphy. With regard to the efficiency of the different diagnostic methods, the authors find a positive predictive value and negative predictive value of $86 \%$ and $95 \%$ for CTA, whereas scintigraphy yields values of $85 \%$ and $96 \%$, respectively (Table 4, PISAPED (1)). It can be assumed that the efficiency of lung scintigraphy would have been even better if ventilation scans had been used instead of chest radiographs. Taking the ALARA principle seriously, the conclusion of this observation should be that lung scintigraphy and CTA show comparable diagnostic efficiency. Because of the lower radiation exposure, the lung scan is the method of choice for the diagnosis of pulmonary embolism. To recommend V/Q scans solely for adolescents and women with large breasts is a merry idea.

The most interesting part of the article is the conclusion. Although it is discussed in depth whether lung scans are favorable in patients older than $50 \mathrm{y}$ or with a shadow on the lung, a blind eye is turned on the efficiency of the PIOPED system itself. To say it with Marcellus, "something is rotten in the state of Denmark." The PISAPED system - and it can hardly be called a system but a simple rule-shows a tremendously higher level of efficiency, and this fact is hardly mentioned. Pardon my saying so, but who cares whether chest radiography can replace the ventilation scan when the real message of the paper is that PIOPED has led the development of lung scintigraphy into a dead-end street. The data clearly indicate that the PISAPED criteria yield better results than the PIOPED system (PIOPED: $72 \%$ positive predictive value, $97 \%$ negative predictive value; PISAPED: $85 \%$ positive predictive value, $96 \%$ negative predictive value). In addition, PISAPED is less complex, is easier to use, and shows a low interobserver variability while minimizing the number of nondiagnostic scans. In several passages of the article it is emphasized that the value of the V/Q scan could be substantially increased by reducing both the number of nondiagnostic examinations and the complexity of the system. Other passages mention that the decline of V/Q scintigraphy is mainly due to the large number of nondiagnostic scans. I fully agree with all these statements. Therefore, the real conclusion of the study is that the PIOPED system cannot stand the test of time. Instead, the PISAPED criteria should be recommended for the interpretation of lung scans because they are highly advantageous with regard to simplicity, reduction of nondiagnostic scans, and diagnostic efficiency. However, the authors are not willing to take this admittedly radical step. Instead, they advise that the PISAPED system should be used only by colleagues with "extensive experience"-a recommendation that is somewhat surprising since the criterion "one or more wedge-shaped perfusion defects" does not sound so terribly complicated to me. If you add "with regular ventilation in the very same anatomic region" you have a fascinatingly simple and highly effective set of criteria to diagnose pulmonary embolism. The decline of the lung scan is a serious loss for nuclear medicine because nuclear medicine has not that much left to lose. Yet, perhaps in this case we may be able to stem the tide by surprising our referring colleagues with definitive and reliable diagnoses.

In Shakespeare's masterpiece, Hamlet asks himself "whether 'tis nobler in the mind to suffer the slings and arrows of outrageous fortune or to take arms against a sea of troubles, and by opposing end them." With regard to the PIOPED system, I recommend the latter.

Patrick Reinartz
Radios-Clinic for Radiology and Nuclear
Medicine
Duesseldorf, Germany

\section{REFERENCES}

1. Sostman HD, Miniati M, Gottschalk A, Matta F, Stein PD, Pistolesi M. Sensitivity and specificity of perfusion scintigraphy combined with chest radiography for acute pulmonary embolism in PIOPED II. J Nucl Med. 2008;49:1741-1748.

2. Reinartz P, Wildberger JE, Schaefer W, Nowak B, Mahnken AH, Buell U. Tomographic imaging in the diagnosis of pulmonary embolism: a comparison between V/Q lung scintigraphy in SPECT technique and multislice spiral CT. J Nucl Med. 2004;45: 1501-1508.

3. Stein PD, Fowler SE, Goodman LR, et al. Multidetector computed tomography for acute pulmonary embolism. N Engl J Med. 2006;354:2317-2327.

4. Howarth DM, Booker JA, Voutnis DD. Diagnosis of pulmonary embolus using ventilation/perfusion lung scintigraphy: more than 0.5 segment of ventilation/ perfusion mismatch is sufficient. Intern Med J. 2006;36:281-288.

5. Brenner DJ, Hall EJ. Computed tomography: an increasing source of radiation exposure. $N$ Engl $J$ Med. 2007;357:2277-2284.

6. Berrington de González A, Darby S. Risk of cancer from diagnostic x-rays: estimates for the UK and 14 other countries. Lancet. 2004;363:345-351. 